\title{
Radiofrequency Ablation and Excision of Multiple Cutaneous Lesions in Neurofibromatosis Type 1
}

\author{
Seong-Hun Kim, Si-Gyun Roh, Nae-Ho Lee, Kyung-Moo Yang \\ Department of Plastic and Reconstructive Surgery, Chonbuk National University Medical School, Jeonju, Korea
}

Background Von Recklinghausen disease or neurofibromatosis type 1 is an autosomal dominant genetic disorder of chromosome 17q11.2. The most common characteristic findings of NF 1 include multiple and recurrent cutaneous neurofibromas associated with psychosocial distress.

Methods Sixteen patients (9 female, 7 male; average age, 31 years; range, 16 to 67 years) with multiple cutaneous neurofibromas between March 2010 and February 2012 were included in the study. All patients were treated with radiosurgical ablation and excision under general anesthesia.

Results All 16 patients were satisfied with the results, when questioned directly during the outpatient department follow-up. The only complaint from a few patients was minimal scarring, but acceptable results were obtained in the end.

Conclusions The radiofrequency procedure is almost bloodless and quick, creating a smaller necrotizing zone. Therefore, instead of employing the time consuming traditional surgery, such as laser therapy and electrosurgical excision, that produces uncertain results and can affect normal adjacent tissue, treatment of neurofibromas with radiofrequency ablation and excision can be an alternative choice of treatment for patients with a large number of neurofibromas.

Keywords Neurofibromatoses / Skin / Radiofrequency / Catheter ablation
Correspondence: Si-Gyun Roh

Department of Plastic and

Reconstructive Surgery, Chonbuk

National University Medical School,

20 Geonji-ro, Deokjin-gu, Jeonju

561-712, Korea

Tel: $+82-63-250-1860$

Fax: +82-63-250-1866

E-mail: pssroh@jbnu.ac.kr

This article was presented at the international meeting, the 11th Japan-Korea Congress of Plastic and Reconstructive Surgery on May 17-19, 2012 in Awaji Island, Japan.

This study was exempted from deliberation by the Institutional Review Board of Chonbuk National University Hospital.

No potential conflict of interest relevant to this article was reported.

\section{INTRODUCTION}

Von Recklinghausen disease or neurofibromatosis type 1 (NF 1) is an autosomal dominant genetic disorder involving chromosome $17 \mathrm{q} 11.2$, and presents as a common heritable neurocutaneous disease. The prevalence of NF1 is approximately 1 in 3,000 live births $[1,2]$. The characteristic findings of NF 1 include caféau-lait spots ( $99 \%$ of all NF 1 patients), the development of plexiform (30\% of all NF 1 patients), and cutaneous neurofibromas (95\% of all NF 1 patients), skinfold freckling, osseous lesions including pseudarthrosis, optic glioma, Lisch nodules in the iris, short stature, and mental retardation [3-6]. In most instances, NF 1 does not develop life-threatening complications, but rather, disfiguring skin tumors are the main burden. Multiple and varying sizes of cutaneous neurofibromas are mainly a condition of cosmetic concern, as their removal is always requested due to the associated psychosocial distress [6]. Various modalities for treatment of cutaneous neurofibromas have been introduced; however, the gold standard for treatment remains controversial.

The radiofrequency $(\mathrm{RF})$ technique has spread worldwide and has been utilized to ablate tumors $[7,8]$. RF tools assemble high energy flux around the electrode tip, thus causing less damage to 
normal tissue around the lesion. The RF technique has several advantages, such as providing an acceptable safety profile for the localized thermal effect without damage to adjacent normal tissue, inducing the hemostasis effect to reduce intraoperative bleeding, and being a relatively quick procedure for small neurofibromas.

In this study, the authors introduce a simple, new RF ablation and excision technique that combines the advantages of a laser technique and a "cold tool," meaning that the radiofrequency procedure is almost bloodless and quick, creating a smaller necrotizing zone [9].

We reviewed the patients' medical records and photographs retrospectively, so this study was exempted from deliberation by the Institutional Review Board of Chonbuk National University Hospital.

\section{METHODS}

Sixteen patients ( 9 female, 7 male; average age, 31 years; range, 16 to 67 years) with multiple cutaneous neurofibromas between March 2010 and February 2012 were included in the study. All of the patients were treated with radiosurgical ablation and excision under general anesthesia and they fulfilled the criteria of the National Institute of Health consensus conference for the diagnosis of NF 1. Most of the patients had one thousand or more neurofibromas concentrated on the trunk with variable sizes ranging from $4 \mathrm{~mm}$ to $10 \mathrm{~cm}$. Six patients had been treated with classical excision therapy and laser therapy in other clinics before. Each patient underwent at least one operation of lesion ablation and excision by the RF surgery unit.

The surgical technique was nearly identical in all cases and done in a sterile operation room. The RF surgical unit (4 MHz radiofrequency Dr. Oppel ST-501, Sometech, Seoul, Korea)

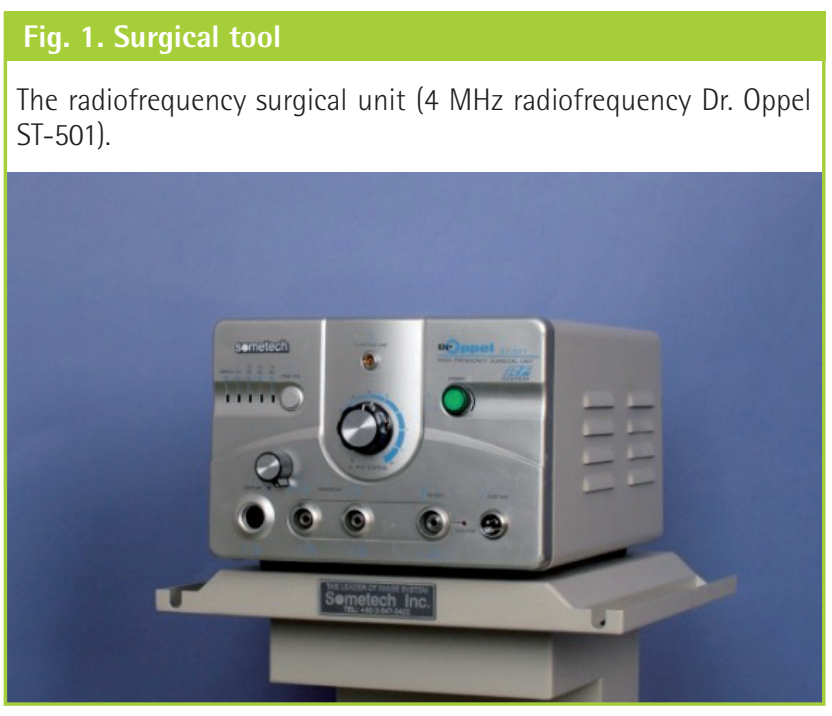

(Fig. 1) was applied as an atraumatic process to cut or ablate the diffuse, nodular, or pedunculated neurofibromas and café-au-lait spots. An approximate frequency of $2.0 \mathrm{MHz}$ is appropriate for ablation, and $4 \mathrm{MHz}$ for cutting. One or two surgeons operated simultaneously with separate hand pieces with needle-point tips (Fig. 2). The short range of the waves ensured that surrounding tissue was not overheated. During the cutting or ablation process, heat disintegrated and vaporized the cells only in the path of the waves. Radiofrequency ablation was directly applied to sessile lesions and café-au-lait spots, essentially vaporizing them. Nodular or pedunculated lesions were grasped with sterile forceps and excised at the base with the radiofrequency needlepoint tip. Skin defects more than $1.0 \mathrm{~cm}$ in diameter were sutured primarily. In such cases, the excised specimen was sent to a pathologist for histopathologic study. Directly after the operation, wound dressing was performed with mupirocin ointment (Bactroban). The wound dressing was replaced daily for 7 days and then only mupirocin ointment (Bactroban) was used twice daily for another 7 to 14 days depending on patient conditions.

All 16 patients were evaluated with the Vancouver scar scale (VSS) and Patient and Observer Scar Assessment Scale (POSAS) in the outpatient department during follow up. The VSS contains 4 variables: vascularity (0-3), pigmentation (0-2), pliability (0-5), and height (0-3). The POSAS include subjective symptoms of pain and pruritus plus the VSS (5-50).

\section{RESULTS}

All 16 patients ( 9 female, 7 male) with multiple cutaneous neurofibromas underwent surgery under general anesthesia. The average duration of surgery was 2 hours and the number of lesions ablated or excised per operation was 80 . The average follow-up period was 11 months. There was no excessive bleed-

\section{Fig. 2. Surgical tool}

A needle-point tip for the hand piece, which is valuable for ablating cutaneous neurofibromas. 
Table 1. Patients and treatment results between March 2010 and February 2012

\begin{tabular}{|c|c|c|c|c|c|c|c|c|}
\hline No. & Sex/Age & $\begin{array}{l}\text { Accompanying } \\
\text { symptoms }\end{array}$ & Comorbidity & Location & $\begin{array}{c}\text { Times of } \\
\text { Op. }\end{array}$ & $\begin{array}{l}\text { No. of lesions } \\
\text { per Op. }\end{array}$ & Complications & $\begin{array}{l}\text { Follow-up } \\
\text { period (mo) }\end{array}$ \\
\hline 1 & $F / 30$ & CAS, SF & & Back, upper ext. & 2 & $73 / 45$ & None & 13 \\
\hline 2 & $\mathrm{M} / 16$ & CAS & & Ant. chest, abdomen & 1 & 57 & None & 5 \\
\hline 3 & $F / 24$ & CAS, SF & & Back, upper ext. & 3 & $92 / 103 / 64$ & None & 19 \\
\hline 4 & $F / 59$ & CAS, SF, Optic glioma & DM & Back & 1 & 135 & None & 3 \\
\hline 5 & $\mathrm{~F} / 67$ & CAS, SF, Plexiform NF & DM, HTN & Back & 1 & 25 & None & 6 \\
\hline 6 & $M / 24$ & CAS, SF & & Face, back & 3 & $17 / 93 / 62$ & None & 20 \\
\hline 7 & $F / 18$ & SF & & Neck, Ant. chest & 1 & 124 & None & 5 \\
\hline 8 & $F / 25$ & CAS, SF & & Back, upper ext. & 2 & $95 / 89$ & None & 15 \\
\hline 9 & $M / 36$ & CAS & & Back & 1 & 48 & None & 4 \\
\hline 10 & $F / 35$ & CAS, SF, Lisch nodule & & Ant. chest, abdomen & 2 & $59 / 124$ & None & 14 \\
\hline 11 & $M / 34$ & CAS, SF & & $\begin{array}{l}\text { Back, upper ext., Ant. } \\
\text { chest, abdomen }\end{array}$ & 4 & $83 / 105 / 72 / 60$ & $\begin{array}{l}\text { Erythema, hyper- } \\
\text { pigmentation scar }\end{array}$ & 28 \\
\hline 12 & $F / 27$ & SF & & Face, upper ext. & 1 & 72 & None & 7 \\
\hline 13 & $M / 25$ & CAS, SF & & Face, upper ext. & 1 & 69 & None & 5 \\
\hline 14 & $F / 38$ & SF, Plexiform NF & & Trunk & 1 & 136 & None & 5 \\
\hline 15 & $\mathrm{M} / 19$ & CAS, SF & & Back, Ant. chest & 3 & $125 / 97 / 58$ & None & 23 \\
\hline 16 & $\mathrm{M} / 26$ & CAS, SF & & Face, Ant. chest & 2 & $95 / 64$ & None & 11 \\
\hline
\end{tabular}

\section{Fig. 3. Case 1}

Case of 23-year-old male with multiple cutaneous lesions on the trunk. (A) Preoperative view. (B) Immediate postoperative view after radiofrequency ablation and excision. (C) Six month follow-up view.
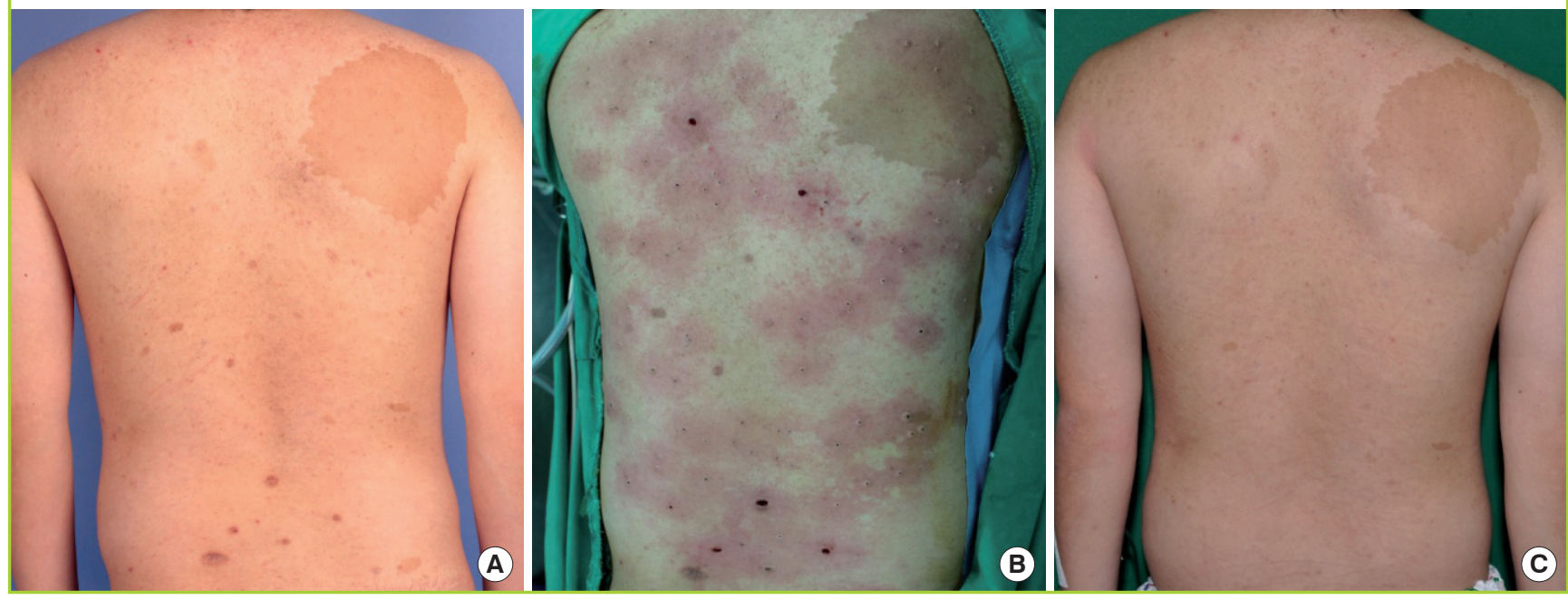

ing, and the operation field was relatively bloodless in all cases. No postoperative complications such as infection, prolonged erythema, or dispigmentation were reported. Re-epithelization time was about 1.5 weeks on the face and 2.5 weeks on the trunk and extremities. Table 1 shows the results of study.

The average VSS score was 6 points and that of the POSAS was 12 points. All 16 patients were satisfied with the results based on being directly questioned in the outpatient department during follow-up. The only complaint from a few patients regarded minimal scarring, but acceptable results were obtained in the end (Figs. 3, 4).

\section{DISCUSSION}

NF 1 is an autosomal dominant genetic disorder of chromosome $17 \mathrm{q} 11.2$ characterized by skin tumors derived from peripheral nerves $[2,10]$. The affected gene is responsible for the production of neurofibromin as a tumor suppressor, possibly through modulation of the oncogenic pathways $[2,10,11]$. Moreover, Schwann cells were discovered to be the origin of neurofibroma, but a more complicated interplay of multiple cell types, such as mast cells and fibroblasts, was observed in tumor genesis, with important implications for surgical treatment of these tumors [12]. 


\section{Fig. 4. Case 2}

Case of 34-year-old female with multiple cutaneous lesions on the trunk. (A) Preoperative view. (B) Immediate postoperative view after radiofrequency ablation and excision. (C) One month follow-up view showing erythematous skin color changes around the wounds due to allergic dermatitis.
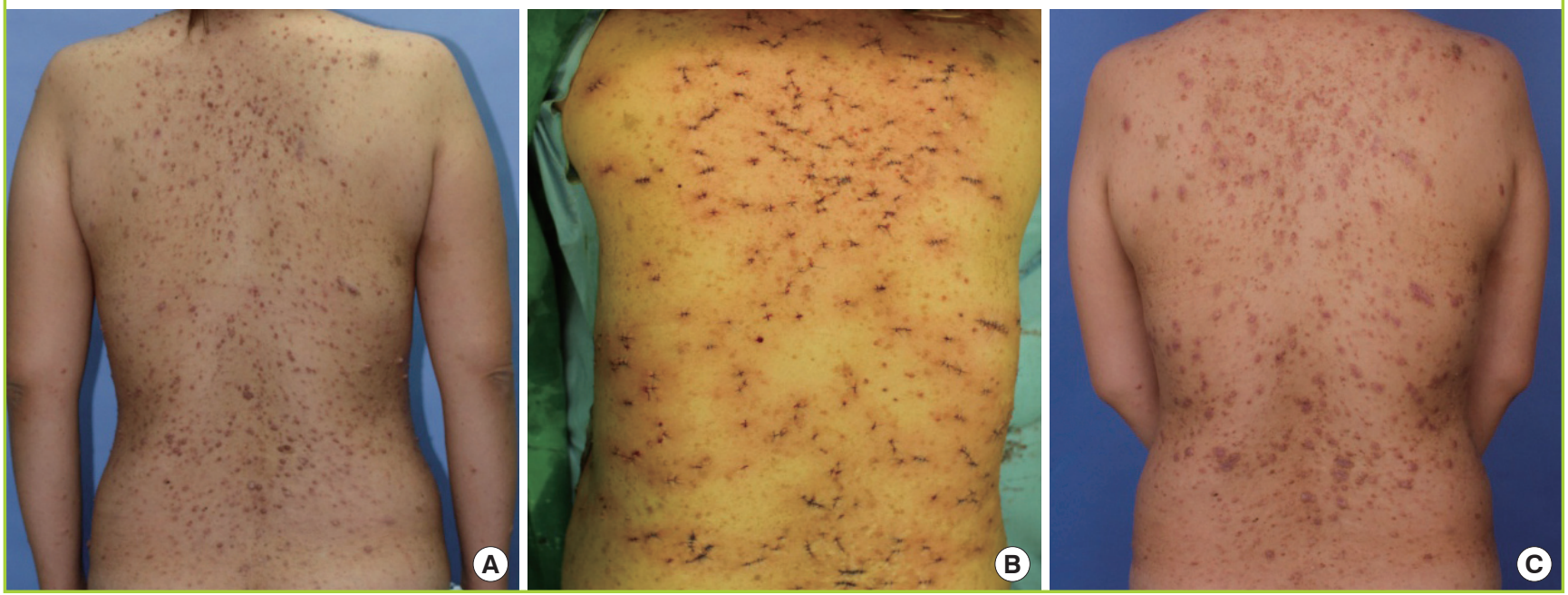

Fig. 5. Scar comparing with surgical and radiofrequency excision

(A) View 1 month postoperatively with surgical excision. (B) View 1 month postoperatively with radiofrequency excision.
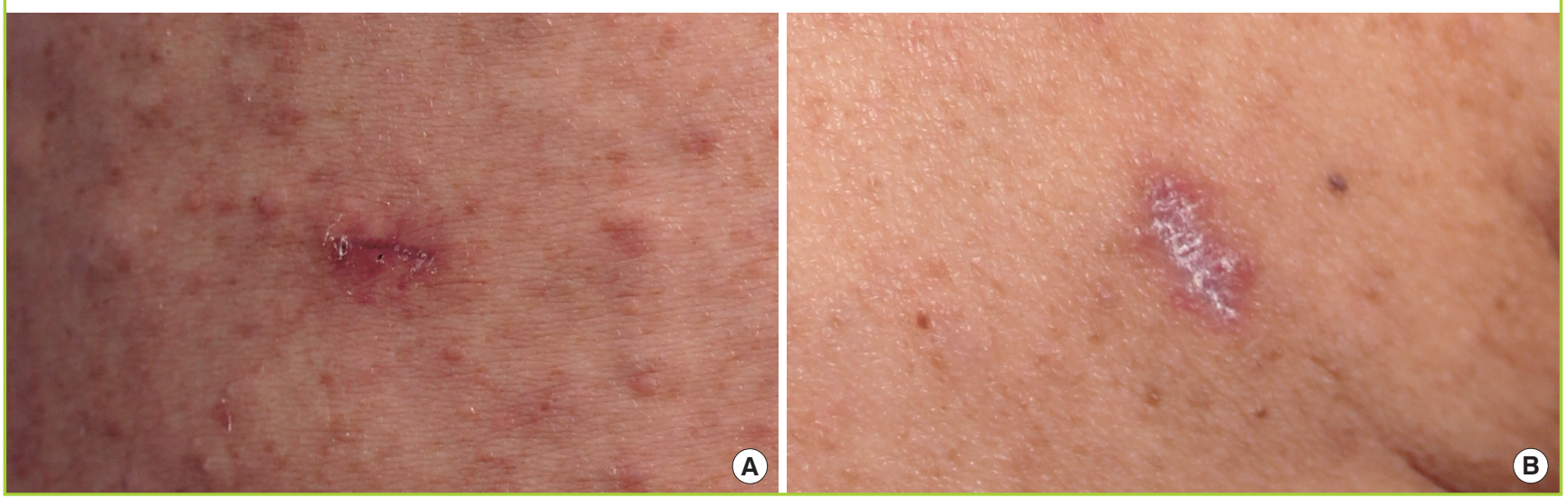

Since one of the most devastating consequences of NF 1 is the psychological impact of the cutaneous lesions, it is the main reason for visiting the hospital.

There are various modalities for treatment of cutaneous neurofibromas; however, none is universally accepted to be the gold standard of treatment. Traditional surgical excision with a scalpel has been used, but it is a time consuming procedure allowing only a small number of neurofibromas to be excised. In addition, radiofrequency excision or ablation causes less scarring than traditional surgical excision (Fig. 5).

Because the size of cutaneous neurofibromas varies from millimeters to centimeters, laser therapy has been used, but it was only useful in removing superficial neurofibromas and caused complications such as hypertrophic scars. Additionally, electro- surgical excision was introduced for the treatment of multiple cutaneous neurofibromas, which was a time sparing process and presented with lower frequencies of intra- and postoperative bleeding but the thermal necrotizing zone of normal tissue was a challenging problem.

Radiofrequency waves as applied in medicine cause thermal ablation of a defined volume of tissue [8]. The RF ablation electrode acts as the cathode of an electrical circuit, which is closed by the application of dispersing pads on the patient's thighs [7]. Because of the small cross-sectional area of the electrode tip, there is a very high energy flux around it. As a result, tissue damage is limited to the part of the circuit that surrounds the electrode tip. However, some disadvantages have been reported, such as skin pad burns and operator-dependent technique. These 
disadvantages can be overcome with careful monitoring of the skin pad site and coaching by senior operators.

Therefore, instead of time consuming traditional surgery with a scalpel, which produces uncertain results or affects normal adjacent tissue, treatment of neurofibromas with radiofrequency ablation and excision can be considered the best choice of treatment for patients with a large number of neurofibromas because of its effectiveness and time saving characteristics.

The critical factor of the surgical procedure is the depth of delamination. The removal of tissue below the depth of the pilosebaceous unit will result in a smooth atrophic scar. Furthermore, an uncertain depth of ablation will result in the recurrence of cutaneous neurofibroma. However, it is not easy to ensure the ablation depth during surgery because hundreds of cutaneous neurofibromas are handled in one operation. Some neurofibroma remnants can exist after incomplete ablation while ablation too deeply can result in smooth atrophic scars. For this reason, careful attention is required for every single ablation or excision.

Despite the need for particular conditions for the best outcome, radiofrequency ablation or excision is easy to learn and relatively simple to perform, requires minimal postoperative care, and provides a satisfactory cosmetic outcome with virtually no complications. The only disadvantage is the high price of the instrument.

This study has the limitation of comparison between traditional excision surgery and radiofrequency ablation due to the lack of pathologic studies. Furthermore, it is uncertain whether radiofrequency ablation can remove cutaneous neurofibromas entirely with a single treatment. The pathologic findings of cutaneous neurofibroma lesions ablated by a radiofrequency unit should be helpful in proving the superiority of radiofrequency ablation.

The most common and characteristic findings of Von Recklinghausen disease or NF 1 are multiple and recurrent cutaneous neurofibromas associated with psychosocial distress. It is a challenge for plastic surgeons to remove as many as thousands of cutaneous neurofibromas with acceptable scars within a limited operation time [13]. The RF technique should be the treatment of choice for ablation and excision of cutaneous neurofibromas since it is almost bloodless and quick, creating a smaller necrotizing zone. RF ablation is likely to remain the mainstay of ablation therapy for a large number of cutaneous neurofibromas until sufficient experience emerges for the widespread acceptance of alternative ablative modalities.

\section{REFERENCES}

1. Huson SM. The neurofibromatoses: classification, clinical features and genetic counseling. In: Kaufmann D, editor. Neurofibromatoses. Basel: Karger; 2008. p. 1-20.

2. Needle MN, Cnaan A, Dattilo J, et al. Prognostic signs in the surgical management of plexiform neurofibroma: the Children's Hospital of Philadelphia experience, 1974-1994. J Pediatr 1997;131:678-82.

3. Riccardi VM. Neurofibromatosis: phenotype, natural history, and pathogenesis. 2nd ed. Baltimore: Johns Hopkins University Press; 1992.

4. Huson SM, Harper PS, Compston DA. Von Recklinghausen neurofibromatosis. A clinical and population study in south-east Wales. Brain 1988;111:1355-81.

5. Listernick R, Louis DN, Packer RJ, et al. Optic pathway gliomas in children with neurofibromatosis 1 : consensus statement from the NF1 Optic Pathway Glioma Task Force. Ann Neurol 1997;41:143-9.

6. Roenigk RK, Ratz JL. $\mathrm{CO}_{2}$ laser treatment of cutaneous neurofibromas. J Dermatol Surg Oncol 1987;13:187-90.

7. Hong K, Georgiades C. Radiofrequency ablation: mechanism of action and devices. J Vasc Interv Radiol 2010;21: S179-86.

8. d'Arsonval MA. Action physiologique des courants alternatifs. C R Seances Soc Biol Fil 1891;43:283-6.

9. Somogyvari K, Battyani Z, Moricz P, et al. Radiosurgical excision of rhinophyma. Dermatol Surg 2011;37:684-7.

10. Yohay KH. The genetic and molecular pathogenesis of NF1 and NF2. Semin Pediatr Neurol 2006;13:21-6.

11. Gottfried ON, Viskochil DH, Fults DW, et al. Molecular, genetic, and cellular pathogenesis of neurofibromas and surgical implications. Neurosurgery 2006;58:1-16.

12. Ablon J. Gender response to neurofibromatosis 1. Soc Sci Med 1996;42:99-109.

13. Becker DW Jr. Use of the carbon dioxide laser in treating multiple cutaneous neurofibromas. Ann Plast Surg 1991;26: 582-6. 